\title{
82. Untersuchung der Antihelminthika. I. Mitteilung Über die wurmabtreibende Wirkung des Alantolactons.
}

\author{
Von Saku Ozeki, Munio Kotake und Kio Hayasi. \\ (Comm. by R. MAJIMA, M.I.A., Oct. 12, 1936.)
}

Seit alters wurde in Japan die Wurzel von Inula Helenium L. als Volksmedizin gebraucht, aber Hansen hat 1931 aus dieser Wurzel Alantolacton, Isoalantolacton und Dihydroalantolacton isoliert und die chemische Konstitution untersucht. Durch diese Untersuchung sowie durch die Untersuchung von Ruzicka ist ihre chemische Konstitution zum grössten Teil festgestellt worden. Es ist ermittelt worden, dass dem Alantolacton eine dem Santonin sehr nahe chemische Konstitution zukommt.<smiles>C=C1C(=O)OC2C1CCC1(C)CCC=C(C)C21</smiles>

Alantolacton<smiles>CCC12C=CC(=O)C(C)=C1C1OC(=O)C(C)C1CC2</smiles>

Santonin

Es lässt sich selbstverständlich denken, dass dieser Körper eine santoninartige, wurmabtreibende Wirkung zeigt. Kariyone hat bereits berichtet, dass es wurmabtreibend wirkt, aber er weist darauf hin, dass es wegen seiner brechenerregenden Wirkung und Bitterkeit nicht angewendet werden kann.

Wir haben diesen Körper sorgfältig weiter untersucht und uns ueberzeugt, dass das von den verunreinigenden, höheren terpenartigen Substanzen vollständig befreite Alantolacton gar nicht brechenerregend wirkt und nur schwach bitter schmeckt, sodass man es durchaus einnehmen kann und, wie die folgende Tabelle zeigt, es ein dem Santonin ueberlegenes Antihelminthicum ist.

\section{Tabelle der letalen Dosis pro $\mathrm{kg}$ Körpergewicht der injizierten Tiere.}

(Zahlen in Klammern bedeuten lebende Stunden)

\begin{tabular}{|c|c|c|c|c|c|}
\hline Tiere & Alan & tolacton & $S a$ & ntonin & Askaridol \\
\hline Maus & 2 & (149) & 0,3 & $(10-15)$ & $0,2 \quad(140-220)$ \\
\hline Ratte & 4 & (126) & 0,2 & (113) & $0,1 \quad(240-300)$ \\
\hline Meerschweinchen & 5 & $(360)$ & & $(32-62)$ & $0,2 \quad(0-92)$ \\
\hline Kaninchen & 0,8 & (158) & 0,5 & $(423)$ & $0,3 \quad(69)$ \\
\hline Hund & 0,3 & $(690)$ & & - & - \\
\hline
\end{tabular}


Ascaris stirbt durch $0,05 \%$ Alantolacton in 20 Stunden, durch $0,1 \%$ in 16 Stunden, aber durch $0,1 \%$ Santonin sind dazu über 2 Tage, durch $0,2 \% 25$ Stunden nötig.

Ausserdem wurde es den Hunden verabreicht und da sich der Urin nicht veränderte, wurde erkannt, dass es bei Tieren schwächer toxisch als Santonin und stark auf Ascaris wirkt. Daher haben die Autoren selbst eingenommen und nachdem sie sich dadurch überzeugt hatten, dass es ungefährlich ist, haben sie es bei Kindern versucht und laut folgender Tabelle sehr gute Erfolge erhalten.

Tabelle der Zahlen der Eierträger nach dem Einnehmen im Vergleich zur Zahlen der Eierträger vor dem Einnehmen.

(In Klammern ist die Dosis)

I. Reihe

\begin{tabular}{c|c|c|c}
\hline Alter & & & \\
$9 \mathrm{j}$. & $0: 3(0,16 \mathrm{~g})$ & $0: 12(0,04 \mathrm{~g})$ & $0: 7(0,02 \mathrm{~g})$ \\
\hline 10, & $0: 1(0,16)$ & $0: 31(0,04)$ & $0: 1(0,02)$ \\
\hline 11, & $0: 1(0,2)$ & $0: 3(0,05)$ & $0: 5(0,025)$ \\
12, & $0: 3(0,2)$ & $0: 15(0,05)$ & $0: 12(0,025)$ \\
\hline
\end{tabular}

Il. Reihe

\begin{tabular}{|c|c|c|c|c|c|}
\hline \multicolumn{6}{|c|}{ Alter } \\
\hline 7 & j. & $2: 11(0,035 \mathrm{~g})$ & $2: 12(0,017 \mathrm{~g})$ & $2: 8 \quad(0,012 \mathrm{~g})$ & $3: 7 \quad(0,009 \mathrm{~g})$ \\
\hline 8 & $"$ & $0: 4 \quad(0,035)$ & $0: 7 \quad(0,017)$ & $0: 12(0,012)$ & $1: 8(0,009)$ \\
\hline 9 & $"$ & $0: 11(0,04)$ & $0: 10(0,020)$ & $0: 7 \quad(0,013)$ & $1: 15 \quad(0,01$ \\
\hline 10 & $"$ & $0: 9 \quad(0,04 \quad)$ & $0: 14(0,020)$ & $0: 11(0,013)$ & $0: 3(0,01)$ \\
\hline 11 & $"$ & $0: 15(0,05)$ & $0: 10(0,025)$ & $0: 5 \quad(0,017)$ & $1: 6 \quad(0,0125)$ \\
\hline 12 & $"$ & $0: 5 \quad(0,05)$ & $0: 4 \quad(0,025)$ & $1: 11(0,025)$ & $1: 8 \quad(0,0125)$ \\
\hline 13 & $"$ & $0: 2 \quad(0,075)$ & $0: 3 \quad(0,04 \quad)$ & $0: 2 \quad(0,025)$ & $1: 2(0,02)$ \\
\hline 14 & $"$ & $0: 1 \quad(0,075)$ & $0: 1 \quad(0,04)$ & $0: 1 \quad(0,025)$ & $0: 1 \quad(0,02)$ \\
\hline
\end{tabular}

Nach unseren obigen Resultaten ist anscheinend nicht mehr nötig, Santonin aus Russland zu importieren. 